KURZ GEMELDET

Allergy News

\section{Die Mutter macht's}

도 gibt einen Zusammenhang zwic schen der Gesamt-IgE-Konzentration im Blut von schwangeren Frauen und dem Risiko für eine atopische Erkrankung des Kindes. Dies stellten taiwanische Wissenschaftler bei der Untersuchung von 545 Elternpaaren vor und nach der Geburt ihrer Kinder fest. Frauen, die vor der Geburt eine GesamtIgE-Konzentration von über $150 \mathrm{kU} / \mathrm{l}$ aufwiesen, hatten häufiger ein Kind mit erhöhter IgE-Konzentration - sowohl im Nabelschnurblut als auch noch 6 Monate nach der Geburt $(\mathrm{p}<0,001)$

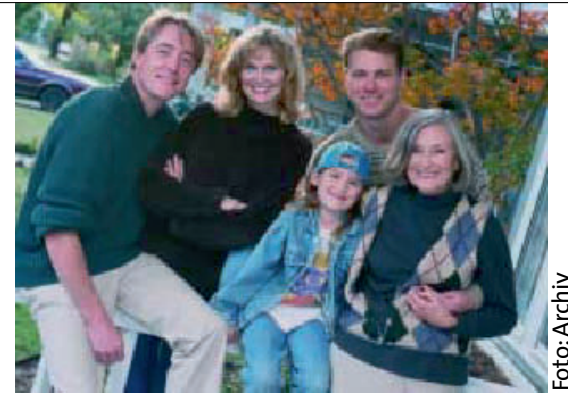

bzw. mit Ekzem. Die Autoren bescheinigen der Bestimmung des mütterlichen Gesamt-IgE eine Spezifität von $83 \%$ und eine Sensitivität von 34\% zur Vorhersage einer atopischen Erkrankung des Kindes. Bei den väterlichen IgE-Konzentrationen fehlte eine Korrelation. $n z$

Liu CA et al. J Allergy Clin Immunol 2003; 112: 899-904

\title{
Unzulängliche Staubprobenanalyse
}

$\mathrm{D}$ ie Belastung von Wohnungen mit Hausstaubmilben und Endotoxinen spielt nicht nur eine wichtige Rolle in epidemiologischen Studien zur Allergiegenese, sondern ist auch Basis für Empfehlungen zur „Wohnungssanierung" bei bereits Erkrankten. Doch spiegeln solche Messungen auch wirklich die exakte Belastung wider? Wohl eher nicht - zumindest wenn die Staubproben nur einmal genommen werden, so das Ergebnis einer deutschen Untersuchung.

\section{Birkenpollen im Februar}

C ine Überraschung erlebten die Aus- werter der Pollenfalle Bochum (Standort: Bergmannsheil) am 3. Februar diesen Jahres, als sie auf Birkenpollen in großer Zahl stießen. Da die Birken in

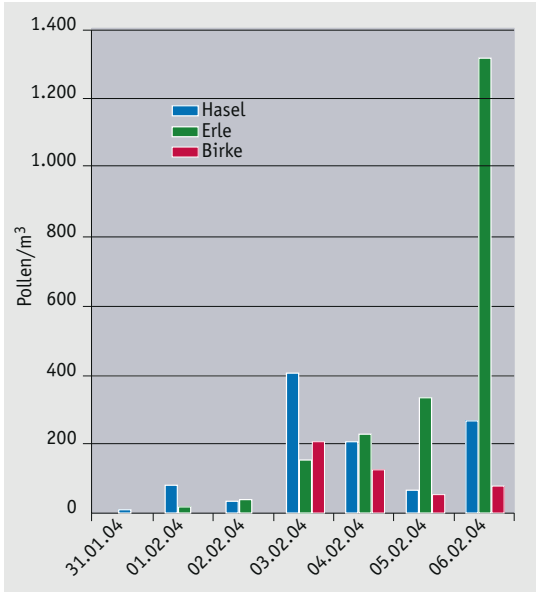

In wiederholten Messungen über 6 Jahre in 152 Haushalten variierten die - im Übrigen sehr geringen - Konzentrationen von Hausstaubmilbenallergenen und Endotoxinen innerhalb einer Wohnung genauso stark wie zwischen den einzelnen Wohnungen. Mehrfachmessungen über einen längeren Zeitraum werden daher dringend angeraten. $\quad b k$

Topp R et al. Clin Exp Allergy 2003; 33: 1659-66

Deutschland auch in klimatisch begünstigten Regionen kaum vor Mitte März ins Blühstadium geraten, kam als Erklärung nur der Ferntransport - wie er z. B. vom Saharasand bekannt ist - in Frage. Dazu passte in der Tat die Wetterlage: Zwischen dem 31. Januar und dem 4. Februar schnellten durch Warmluftzufuhr auf der Vorderseite eines atlantischen Tiefs die Temperaturmaxima von knapp über 0 auf $16-19^{\circ} \mathrm{C}$ nach oben. Es kam es zu der in der Grafik gezeigten Belastung durch Hasel- und Erlenpollen. Zusätzlich blies in rund $1.500 \mathrm{~m}$ Höhe ein kräftiger Südwestwind, der mit 75 bis $110 \mathrm{~km} / \mathrm{h}$ nicht nur die milde Biskayaluft, sondern auch Birkenpollen aus Südwestfrankreich mit sich brachte.

Dr. Gunild Scheid, Deutscher Wetterdienst Essen

\section{Strahlender Vergleich}

ie Nutzung eines Mobiltelefons hat eine Belastung des Kopfes mit hochfrequenter elektromagnetischer Strahlung zur Folge, die mit Hilfe der spezifischen Absorptionsrate (SAR) quantifiziert wird. Eine SAR von unter $2 \mathrm{~W} / \mathrm{kg}$ ist nach Einschätzung der Strahlenschutzkommission unbedenklich und zugleich die Voraussetzung, dass ein Mobiltelefon in Deutschland vertrieben werden darf. Während einige der auf dem Markt befindlichen Handys, so das Ergebnis einer Messreihe des Bundesamtes für Strahlenschutz vom November 2003, diesen Grenzwert

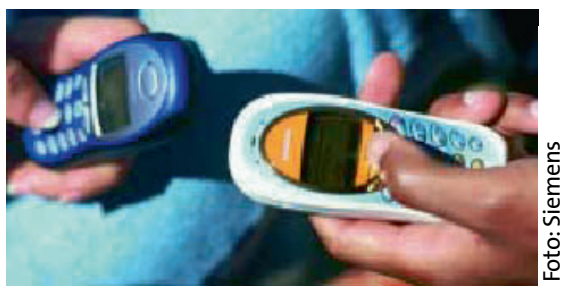

eher knapp unterschreiten (Samsung SGH-Q200: 1,45 W/kg), liegen andere Geräte bis zu drei Zehnerpotenzen darunter (LG G7000: 0,005 W/kg). Die komplette Liste des Bundesamtes für Strahlenschutz mit den SAR-Werten von 236 Mobiltelefonen ist im Internet abrufbar.

www.bfs.de/elektro/hff/oekolabel.html

\section{Zweitmeinung gefragt}

aut einer Studie der Boston Consu— ting Group über das Web-Verhalten europäischer Mediziner suchen 51\% aller ärztlichen Nutzer Rat und Austausch mit Fachkollegen im Internet. Bei www.multimedica.de, dem großen Ärzte-Online-Dienst aus dem Hause Springer, können sich Kollegen nicht nur untereinander austauschen, sondern in kniffligen Fällen auch jederzeit ausgewählte Spezialisten eines Fachbereiches zu Rate ziehen. Im Moment gibt es fast 50 dieser interaktiven Expertenräte. Der Service ist für registrierte Nutzer kostenfrei.

red

www.multimedica.de/expertenrat.htm 